October 1, 2018

\title{
CASIMIR EFFECTS NEAR THE BIG RIP SINGULARITY IN VISCOUS COSMOLOGY
}

\author{
Iver Brevik ${ }^{1 *}$, Olesya Gorbunova ${ }^{2 \dagger}$, Diego Sáez-Gómez ${ }^{3 \ddagger}$ \\ ${ }^{1}$ Department of Energy and Process Engineering, \\ Norwegian University of Science and Technology, N-7491 Trondheim, Norway \\ ${ }^{2}$ Tomsk State Pedagogical University, Tomsk, Russia and \\ ${ }^{3}$ Institut de Ciencies de l'Espai (IEEC-CSIC), E-08193 Bellaterra (Barcelona), Spain
}

\begin{abstract}
Analytical properties of the scalar expansion in the cosmic fluid are investigated, especially near the future singularity, when the fluid possesses a constant bulk viscosity $\zeta$. In addition, we assume that there is a Casimir-induced term in the fluid's energy-momentum tensor, in such a way that the Casimir contributions to the energy density and pressure are both proportional to $1 / a^{4}, a$ being the scale factor. A series expansion is worked out for the scalar expansion under the condition that the Casimir influence is small. Close to the Big Rip singularity the Casimir term has however to fade away and we obtain the same singular behavior for the scalar expansion, the scale factor, and the energy density, as in the Casimir-free viscous case.

PACS numbers: 98.80.-k, 95.36.+x
\end{abstract}

\section{INTRODUCTION}

From a hydrodynamical viewpoint it is almost surprising to notice that the cosmic fluid - whether considered in the early or in the late epochs - is usually taken to be nonviscous. After all, there are two viscosity (shear and bulk) coefficients naturally occurring in general linear hydrodynamics; the linear approximation meaning physically that one is considering only first order deviations from thermal equilibrium. The shear viscosity coefficient is evidently of importance when dealing with flow near solid surfaces, but it can be crucial also under boundary-free conditions such as in isotropic turbulence. In later years it has become more common to take into account viscosity properties of the cosmic fluid, however. Because of assumed spatial isotropy in the fluid the shear viscosity is usually left out; any anisotropic deviations like those encountered in the Kasner universe become rather quickly smoothed out. Thus only the bulk viscosity coefficient, called $\zeta$, remains in the energy-momentum tensor of the fluid. One should here note, however, that at least in the plasma region in the early universe the value of the shear viscosity as derived from kinetic theory is greater than the bulk viscosity by many orders of magnitude. Cf., for instance, Refs. [1] and [2].

Early treatises on viscous cosmology are given by Padmanabhan and Chitre [3] and Grøn [4], the latter paper being an extensive review of the field up to 1990 . We have ourselves dealt earlier with viscous entropy production in the early universe [2], and viscous fluids on the Randall-Sundrum branes [5, 6]. Cataldo et al. considered viscous dark energy and phantom evolution using Eckhart's theory of irreversible thermodynamics 7]. Kofinas et al. considered the crossing of the $w=-1$ barrier using a brane-bulk energy exchange model with an induced gravity curvature correction [8]. As discussed by Nojiri and Odintsov [9] and by Capozziello et al. [10], a dark fluid with a time dependent bulk viscosity can be considered as a fluid with an inhomogeneous equation of state. Some other recent papers on viscous cosmology are Refs. [11, 12, 13].

A special branch of viscous cosmology is to investigate how the bulk viscosity can influence the future singularity, commonly called the Big Rip, when the fluid is in the phantom state corresponding to the thermodynamic parameter $w$ being less than -1 . Some recent papers in this direction are Refs. 14, 15, 16, 17, 18, 19, 20, 21]. In particular, as first pointed out in Ref. [14], the presence of a bulk viscosity proportional to the scalar expansion $\theta$ can cause the fluid to pass from the quintessence region into the phantom region and thereby inevitably lead to a future singularity.

The purpose of the present paper is to generalize these viscous cosmology theories in the sense that we take into account the Casimir effect. We shall model the Casimir influence by writing the total Casimir energy in the same form as for a perfectly conducting shell, identifying the cosmological "shell" radius essentially with the cosmic scale factor. This is a very simple, though natural, approach to the problem. The approach is of the same kind as that followed in an earlier quantum cosmology paper dealing with the expanding FRW universe in the nonviscous case

\footnotetext{
* E-mail:iver.h.brevik@ntnu.no

$\dagger$ E-mail:gorbunovaog@tspu.edu.ru

‡ E-mail: saez@ieec.uab.es
} 
[22]. We mention that there are also other ways of treating the influence from the Casimir effect in cosmology; the reader may consult, for instance, Refs. [23, 24, 25, 26, 27].

As bulk viscosity corresponds to inhomogeneous Hubble rate dependent terms in the effective equation of state, the situation is quite similar to that of modified gravity theory. For a general treatise on modified gravity, see the review of Nojiri and Odintsov [28].

\section{FORMALISM, WHEN THE CASIMIR EFFECT IS OMITTED}

We start with the standard FRW metric,

$$
d s^{2}=-d t^{2}+a^{2}(t) d \mathbf{x}^{2}
$$

and set the spatial curvature $k$, as well as the cosmological constant $\Lambda$, equal to zero. We let subscript 'in' refer to present time quantities, and choose $t_{i n}=0$. The scale factor $a(t)$ is normalized such that $a(0) \equiv a_{i n}=1$. The equation of state is taken as

$$
p=w \rho,
$$

with $w$ constant. As mentioned, $w<-1$ in the phantom region (ordinary matter is not included in the model). The bulk viscosity $\zeta$ is taken to be constant. The energy-momentum tensor of the fluid can be expressed as

$$
T_{\mu \nu}=\rho U_{\mu} U_{\nu}+\tilde{p}\left(g_{\mu \nu}+U_{\mu} U_{\nu}\right)
$$

where $U_{\mu}$ is the comoving four-velocity and $\tilde{p}=p-\zeta \theta$ is the effective pressure, $\theta=3 H=3 \dot{a} / a$ being the scalar expansion. The Friedmann equations take the form

$$
\begin{gathered}
\theta^{2}=24 \pi G \rho, \\
\dot{\theta}+\frac{1}{6} \theta^{2}=-4 \pi G \tilde{p} .
\end{gathered}
$$

The energy conservation equation leads to

$$
\dot{\rho}+(\rho+p) \theta=\zeta \theta^{2}
$$

From the above equations the differential equation for the scalar expansion, with $w$ and $\zeta$ as free parameters, can be derived as

$$
\dot{\theta}+\frac{1}{2}(1+w) \theta^{2}-12 \pi G \zeta \theta=0 .
$$

The solution is, when subscript zero signifies that the Casimir effect is so far left out,

$$
\theta_{0}(t)=\frac{\theta_{i n} e^{t / t_{c}}}{1+\frac{1}{2}(1+w) \theta_{i n} t_{c}\left(e^{t / t_{c}}-1\right)} .
$$

Here $\theta_{i n}$ is the initial (present-time) scalar expansion and $t_{c}$ the 'viscosity time'

$$
t_{c}=\frac{1}{12 \pi G \zeta} .
$$

Since $(1+w)<0$ by assumption, it follows from Eq. (8) that the future singularity occurs at a rip time $t_{s 0}$ given by

$$
t_{s 0}=t_{c} \ln \left[1-\frac{2}{1+w} \frac{1}{\theta_{i n} t_{c}}\right] .
$$

This means that in the nonviscous limit $\zeta \rightarrow 0$,

$$
t_{s 0}=-\frac{2}{1+w} \frac{1}{\theta_{i n}},
$$

which is independent of $\zeta$. At the other extreme, in the high viscosity limit $t_{c} \rightarrow 0$,

$$
t_{s 0}=t_{c} \ln \left[\frac{-2}{1+w} \frac{1}{\theta_{i n} t_{c}}\right],
$$

showing that $t_{s 0}$ becomes small. The fluid is quickly driven into the Big Rip singularity. 


\section{THE CASIMIR EFFECT INCLUDED}

As mentioned above, a simple and natural way of dealing with the Casimir effect in cosmology is to relate it to the single length parameter in the $(k=0)$ theory, namely the scale factor $a$. It means effectively that we should put the Casimir energy $E_{c}$ inversely proportional to $a$. This is in accordance with the basic property of the Casimir energy, viz. that it is a measure of the stress in the region interior to the "shell" as compared with the unstressed region on the outside. The effect is evidently largest in the beginning of the universe's evolution, when $a$ is small. At late times, when $a \rightarrow \infty$, the Casimir influence should be expected to fade away. As we have chosen $a$ nondimensional, we shall introduce an auxiliary length $L$ in the formalism. Thus we adopt in model in which

$$
E_{c}=\frac{C}{2 L a},
$$

where $C$ is a nondimensional constant. This is the same form as encountered for the case of a perfectly conducting shell [29]. In the last-mentioned case, $C$ was found to have the value

$$
C=0.09235
$$

The expression (13) is of the same form as adopted in Ref. 22] (cf. also [30]). It is strongly related to the assumptions made by Verlinde when dealing with the holographic principle in the universe [31]. Cf. also the papers [32] and [33] dealing with the holographic principle applied to viscous cosmology.

In the following we shall for definiteness assume $C$ to be positive, corresponding to a repulsive Casimir force, though $C$ will not necessarily be required to have the value (14). The repulsiveness is a characteristic feature of conducting shell Casimir theory, following from electromagnetic field theory under the assumption that dispersive short-range effects are left out ([29]; cf. also [30]). Another assumption that we shall make, is that $C$ is small compared with unity. This is physically reasonable, in view of the conventional feebleness of the Casimir force.

The expression (13) corresponds to a Casimir pressure

$$
p_{c}=\frac{-1}{4 \pi(L a)^{2}} \frac{\partial E_{c}}{\partial(L a)}=\frac{C}{4 \pi L^{4} a^{4}}
$$

and leads consequently to a Casimir energy density $\rho_{c} \propto 1 / a^{4}$.

The Casimir energy-momentum tensor

$$
T_{\mu \nu}^{c}=\rho_{c} U_{\mu} U_{\nu}+p_{c}\left(g_{\mu \nu}+U_{\mu} U_{\nu}\right)
$$

together with the Casimir equation of state $p_{c}=w_{c} \rho_{c}$, yield the energy balance

$$
\frac{\dot{\rho}_{c}}{\rho_{c}}+\left(1+w_{c}\right) \theta=0,
$$

having the solution $\rho_{c} a^{3\left(1+w_{c}\right)}=$ constant. To get $\rho_{c} \propto 1 / a^{4}$ we must have $w_{c}=1 / 3$. The Casimir contributions to the pressure and energy density become accordingly

$$
p_{c}=\frac{C}{8 \pi L^{4} a^{4}}, \quad \rho_{c}=\frac{3 C}{8 \pi L^{4} a^{4}} .
$$

The Friedmann equations now become

$$
\begin{gathered}
\theta^{2}=24 \pi G\left(\rho+\frac{3 C}{8 \pi L^{4} a^{4}}\right), \\
\dot{\theta}+\frac{1}{6} \theta^{2}=-4 \pi G\left(w \rho-\zeta \theta+\frac{C}{8 \pi L^{4} a^{4}}\right),
\end{gathered}
$$

while the energy conservation equation preserves its form,

$$
\dot{\rho}+(1+w) \rho \theta=\zeta \theta^{2}
$$

Note again that we are considering the dark energy fluid only, with density $\rho$ and thermodynamical parameter $w$. The ordinary matter fluid (dust) is left out. 
Solving $\rho$ from Eq. (19) and inserting into Eq. (21) we obtain as governing equation for the scalar expansion

$$
\dot{\theta}+\frac{1}{2}(1+w) \theta^{2}-12 \pi G \zeta \theta=-(1-3 w) \frac{3 G C}{2 L^{4} a^{4}} .
$$

It is convenient to introduce the constant $\alpha$, defined as

$$
\alpha=-(1+w)>0
$$

and also to define the quantity $X(t)$,

$$
X(t)=1-\frac{1}{2} \alpha \theta_{i n} t_{c}\left(e^{t / t_{c}}-1\right)
$$

which satisfies

$$
X(0)=1, \quad X\left(t_{s 0}\right)=0
$$

In view of the assumed smallness of $C$ we now make a Stokes expansion for $\theta$ to the first order,

$$
\theta(t)=\theta_{0}(t)+C \theta_{1}(t)+O\left(C^{2}\right)
$$

using henceforth the convention that subscript zero refers to the $C=0$ case. The zeroth order solution is

$$
\theta_{0}(t)=\theta_{i n} X^{-1} e^{t / t_{c}}
$$

in accordance with Eq. (8). It corresponds to the zeroth order scale factor

$$
a_{0}(t)=X^{-\frac{2}{3 \alpha}}
$$

satisfying $a_{0}(0)=1$ as before.

As the right hand side of Eq. (22) is already of order $C$, we can replace $a(t)$ with $a_{0}(t)$ in the denominator. Thus we get the following equation for the first order correction coefficient $\theta_{1}$ :

$$
\dot{\theta}_{1}-\left(\alpha \theta_{i n} X^{-1} e^{t / t_{c}}+12 \pi G \zeta\right) \theta_{1}=-(1-3 w) \frac{3 G}{2 L^{4}} X^{\frac{8}{3 \alpha}}
$$

We impose the same initial condition for the scalar expansion as in the $C=0$ case: $\theta(0)=\theta_{0}(0) \equiv \theta_{\text {in }}$. It means according to Eq. (26) that $\theta_{1}(0)=0$.

The homogeneous solution of Eq. (29), called $\theta_{1 h}$, may be written

$$
\theta_{1 h}(t)=\exp \left[\int_{0}^{t}\left(\alpha \theta_{i n} X^{-1} e^{t / t_{c}}+12 \pi G \zeta\right) d t\right]
$$

satisfying $\theta_{1 h}(0)=1$. The the full solution becomes

$$
\theta_{1}(t)=-(1-3 w) \frac{3 G}{2 L^{4}} \theta_{1 h}(t) \cdot \int_{0}^{t} \frac{X^{\frac{8}{3 \alpha}}}{\theta_{1 h}} d t
$$

satisfying $\theta_{1}(0)=0$. The two terms on the right hand side of Eq. (26),$\theta_{0}(t)$ and $C \theta_{1}(t)$, are accordingly determined.

\section{A. Analytic approximation for low viscosity}

Although in general the expression for $\theta(t)$ has to be calculated numerically, the main features of the solution can be shown already analytically. Consistent with the assumed smallness of $C$ we need not distinguish between the rip time $t_{s}$ corresponding to $C \neq 0$ and the rip time $t_{s 0}$ corresponding to $C=0$. Let us assume for mathematical simplicity the low-viscosity limit

$$
\theta_{i n} t_{c} \gg 1
$$


being physically the most important case also. It corresponds to $t_{s 0} / t_{c}=2 /\left(\alpha \theta_{i n} t_{c}\right) \ll 1$. Then,

$$
\begin{gathered}
X(t) \sim \frac{1}{2} \alpha \theta_{i n}\left(t_{s 0}-t\right)=\frac{t_{s 0}-t}{t_{s 0}}, \\
\theta_{0}(t) \sim \frac{2}{\alpha} \frac{1}{t_{s 0}-t}, \\
a_{0}(t) \sim\left(\frac{t_{s 0}}{t_{s 0}-t}\right)^{\frac{2}{3 \alpha}} .
\end{gathered}
$$

Both $\theta_{0}(t)$ and $a_{0}(t)$ diverge $\left(\alpha>0\right.$ by assumption). Using Eq. (33) we can calculate $\theta_{1}(t)$ from Eq. (31),

$$
\theta_{1}(t)=-(1-3 w) \frac{9 G}{2 L^{4}} \frac{\alpha t_{s 0}}{8+9 \alpha} \frac{1-\left(1-t / t_{s 0}\right)^{\frac{8}{3 \alpha}+3}}{\left(1-t / t_{s 0}\right)^{2}}
$$

From the expansion (26) we thus obtain for the scalar expansion to the first order in $C$,

$$
\theta(t)=\frac{2}{\alpha t_{s 0}} \frac{1}{1-t / t_{s 0}}\left\{1-(1-3 w) \frac{9 G C}{4 L^{4}} \frac{\alpha^{2} t_{s 0}^{2}}{8+9 \alpha} \frac{1-\left(1-t / t_{s 0}\right)^{\frac{8}{3 \alpha}}+3}{1-t / t_{s 0}}\right\}
$$

The viscosity is absent in this expression. This is as we would expect in view of the low-viscosity approximation.

The expression (37) cannot, however, be valid near the singularity. The reason is that the Taylor expansion in $C$ in Eq. (26) is not applicable at $t=t_{s 0}$. The solution (37) can be applied safely as long as $t$ stays considerably smaller than $t_{s 0}$. By making a first order expansion in $t / t_{s 0}$ of the expression between the curly parentheses we can write the solution in simplified form as

$$
\theta(t)=\frac{2}{\alpha t_{s 0}} \frac{1}{1-t / t_{s 0}}\left\{1-(1-3 w) \frac{3 G C \alpha t_{s 0}}{4 L^{4}} t\right\}, \quad t \ll t_{s 0} .
$$

As $(1-3 w)>0$ this means that $\theta(t)$ becomes slightly reduced by the Casimir term. The repulsive Casimir force causes the energy density $\rho$ in Eq. (19) to be slightly smaller than in the $C=0$ case.

To deal with the conditions close to the singularity, we have to go back to the governing equations themselves.

\section{B. Behavior close to the singularity}

To begin with, it is instructive to list the general classification of possible future singularities as given in Refs. 34] and [21]. If $t_{s}$ denotes the rip time, one has four types,

(i) Type I (original "Big Rip"): For $t \rightarrow t_{s}, a \rightarrow \infty, \rho \rightarrow \infty$, and $|p| \rightarrow \infty$, or $p$ and $\rho$ are finite at $t=t_{s}$.

(ii) Type II ("sudden"): For $t \rightarrow t_{s}, a \rightarrow a_{s}, \rho \rightarrow \rho_{s}$, and $|p| \rightarrow \infty$,

(iii) Type III: For $t \rightarrow t_{s}, a \rightarrow a_{s}, \rho \rightarrow \infty$, and $|p| \rightarrow \infty$,

(iv) Type IV: For $t \rightarrow t_{s}, a \rightarrow a_{s}, \rho \rightarrow 0,|p| \rightarrow 0$, or $p$ and $\rho$ are finite. Higher order derivatives of $H$ diverge.

The singularities we have been contemplating above are seen to be of Type I. As $a \rightarrow \infty$ near the singularity, we can draw the important conclusion from Friedmann's equations (19) and (20) that the influence from the Casimir term fades away. Close to the singularity we simply obtain the same solutions as in the Casimir-free case.

Let now $t_{\zeta s}$ denote the singularity time in the presence of viscosity. We thus get

$$
t_{s \zeta}=t_{c} \ln \left(1+\frac{2}{\alpha} \frac{1}{\theta_{\text {in }} t_{c}}\right) .
$$

It corresponds to $\theta\left(t_{s \zeta}\right)=\infty$. We see that $t_{s \zeta}$ is always less than the singularity time for the nonviscous case,

$$
t_{s \zeta}<t_{s, \zeta=0}=\frac{2}{\alpha \theta_{i n}} .
$$


For the scalar expansion we find close to the singularity, again assuming for simplicity low viscosity so that $\theta_{\text {in }} t_{c} \gg 1$ [18],

$$
\theta(t)=\frac{2 / \alpha}{t_{s \zeta}-t}, \quad t \rightarrow t_{s \zeta}
$$

In turn, this corresponds to

$$
\begin{gathered}
a(t) \sim \frac{1}{\left(t_{s \zeta}-t\right)^{2 / 3 \alpha}}, \quad t \rightarrow t_{s \zeta}, \\
\rho(t) \sim \frac{1}{\left(t_{s \zeta}-t\right)^{2}}, \quad t \rightarrow t_{s \zeta} .
\end{gathered}
$$

\section{On the nonviscous case}

It may finally be worthwhile to consider the entirely nonviscous case, while keeping $C>0$. Setting $\zeta=0$ we get from Eq. (22) the governing equation for the scale factor $a$ :

$$
a^{3} \ddot{a}+\frac{1}{2}(1+3 w) a^{2} \dot{a}^{2}=-(1-3 w) \frac{G C}{2 L^{4}} .
$$

This equation is still not solvable analytically. We get for $X(t), \theta_{0}(t)$ and $a_{0}(t)$ the same expressions as in Eqs. (33) (35). Similarly, we get for $\theta(t)$ the same expansion (37) as before, with $t_{s 0}=2 /\left(\alpha \theta_{i n}\right)$. These results are as expected, in view of the property of continuity with respect to variation of parameters.

\section{CONCLUDING REMARKS}

Considerable attention has recently been devoted to the behavior of the dark energy fluid near the future singularity. Various possibilities have been contemplated. In addition to the references given above [23] -[27], we may refer also to the papers [35]. It has even been suggested that the future singularity can be avoided via quantum gravity effects. Thus in Ref. [36] it is shown how the universe may turn into a de Sitter phase.

Let us finally summarize our results above:

1) If $\zeta>0$ and $C=0$, the rip singularity time $t_{s 0}$ is given by Eq. (10). In particular, if $\zeta \rightarrow 0$ then Eq. (111) holds.

2) If $\zeta>0$ and $C>0$, the scalar expansion $\theta(t)$ is given by the first-order series (26), with $\theta_{0}(t)$ and $\theta_{1}(t)$ given by Eqs. (27) and (31). In the low-viscosity case $\theta_{i n} t_{c} \gg 1, \theta(t)$ is given by the series (37) which, however, is not applicable near the singularity as $\theta(t)$ is not analytic in $C$ at the singularity.

3) Near the singularity, the Casimir effect fades away and the viscous rip time $t_{s \zeta}$ is given by Eq. (39). Corresponding values for $\theta(t), a(t)$ and $\rho(t)$ near the singularity follow from Eqs. (41) - (43).

4) If $\zeta=0$ and $C>0$, the governing equation for $a(t)$ is Eq. (44).

\section{Acknowledgment}

O. Gorbunova acknowledges support from the ESF Programme "New Trends and Applications of the Casimir Effect" and by Grant the Scientific School LRSS Project N.2553.2008.2. D. Saez-Gomez acknowledges a grant from MICINN (Spain), project FIS2006-02842, DSG's research was performed while on leave at Department of Physics of NTNU (Norway)

[1] N. Caderni and R. Fabbri, Phys. Lett. 69B, 508 (1977).

[2] I. Brevik and L. T. Heen, Astrophys. Space Sci. 219, 99 (1994).

[3] T. Padmanabhan and S. M. Chitre, Phys. Lett. A 120, 433 (1987).

[4] Ø. Grøn, Astrophys. Space Sci. 173, 191 (1990). 
[5] I. Brevik and A. Hallanger, Phys. Rev. D 69, 024009 (2004).

[6] I. Brevik, J. M. Børven and S. Ng, Gen. Relativ. Gravit. 38, 907 (2006).

[7] M. Cataldo, N. Cruz and S. Lepe, Phys. Lett. B 619, 5 (2005).

[8] G. Kofinas, G. Panotopoulos and N. Tomaras, JHEP 0601, 107 (2006).

[9] S. Nojiri and S. D. Odintsov, Phys. Rev. D 72, 023003 (2005).

[10] S. Capozziello, V. F. Cardone, E. Elizalde, S. Nojiri and S. D. Odintsov, Phys. Rev. D 73, 043512 (2006).

[11] B. Li and J. D. Barrow, arXiv:0902.3163 [gr-qc].

[12] J. Chen and Y. Wang, arXiv:0904.2808 [gr-qc].

[13] C. J. Feng and Xin-Zhou Li, arXiv:0905.0527 [astro-ph.CO].

[14] I. Brevik and O. Gorbunova, Gen. Relativ. Gravit. 37, 2039 (2005).

[15] I. Brevik, O. Gorbunova and Y. A. Shaido, Int. J. Mod. Phys. D 14, 1899 (2005).

[16] I. Brevik, Gen. Rel. Gravit. 38, 1317 (2006).

[17] I. Brevik, Gravitation and Cosmology 14, 332 (2008).

[18] I. Brevik and O. Gorbunova, Eur. Phys. J. C 56, 425 (2008).

[19] I. Brevik, Eur. Phys. J. C 56, 579 (2008).

[20] Ø. Grøn, in The Casimir Effect and Cosmology: A volume in honour of Professor Iver H. Brevik on the occasion of his 70th birthday, Editors S. D. Odintsov, E. Elizalde and O. G. Gorbunova (Tomsk State Pedagogical University Press, 2008), p. 75 ; arXiv:0812.2549.

[21] I. Brevik and O. Gorbunova, in The Problems of Modern Cosmology: A volume in honour of Professor S. D. Odintsov on the occasion of his 50th birthday, Editor P. M. Lavrov (Tomsk State Pedagogical University Press, 2009), p. 106; arXiv:0811.1129.

[22] I. Brevik, K. A. Milton, S. D. Odintsov and K. E. Osetrin, Phys. Rev. D 62, 064005 (2000).

[23] W. Godlowski, M. Szydlowski and Z. H. Zhu, Gravitation and Cosmology 11, 1 (2005).

[24] M. Schaden, Phys. Rev. A 73, 042102 (2006).

[25] B. P. Dolan and C. Nash, Commun. Math. Phys. 148, 139 (1992).

[26] E. Elizalde, S. Nojiri, S. D. Odintsov and S. Ogushi, Phys. Rev. D 67, 063515 (2003).

[27] E. Elizalde, J. Phys. A 39, 6299 (2006).

[28] S. Nojiri and S. D. Odintsov, Int. J. Geom. Meth. Mod. Phys. 4, 115 (2007) arXiv:hep-th/0601213.

[29] K. A. Milton, L. L. DeRaad, Jr. and J. Schwinger, Ann. Phys. (N.Y.) 115, 388 (1978).

[30] I. Brevik, B. Jensen and K. A. Milton, Phys. Rev. D 64, 088701 (2001).

[31] E. Verlinde, arXiv:hep-th/0008140

[32] I. Brevik and S. D. Odintsov, Phys. Rev. D 65, 067302 (2002).

[33] I. Brevik, Phys. Rev. D 65, 127302 (2002).

[34] S. Nojiri, S. D. Odintsov and S. Tsujikawa, Phys. Rev. D 71, 063004 (2005).

[35] R. R. Caldwell, M. Kamionkowski and N. N. Weinberg, Phys. Rev. Lett. 91, 071301 (2003); S. Nojiri and S. D. Odintsov, Phys. Lett. B 562, 147 (2003).

[36] E. Elizalde, S. Nojiri and S. D. Odintsov, Phys. Rev. D 70, 043539 (2004). 